Comparing this with the results obtained by the old process and recorded in Table $I$, a saving of 0.20 gallon alcohol, 94 per cent., and 4 I 5 lbs. bleaching powder, 35 per cent., was effected for every roo lbs, of chloroform made by the new process.

The saving in alcohol was insignificant, but there was no saving expected, the experiments indicating the consumption of II.5 gallons alcohol for every I00 lbs. of chloroform, while i i.53 gallons were used in actual work. The gain in bleaching powder, however, was 29 per cent., quite near to the 34 per cent. saving, which were indicated by the most favorable experiments in Series N. The consumption of steam was larger than in the old process, since about 400 gallons diluted alcohol were to be distilled off in each operation, in order to recover all the alcohol. About 3 tons steam were required for each apparatus in a day's work. However, I500 1bs. bleach could be worked daily in each of the six stills, in place of 1000 lbs., and $147 \mathrm{lbs}$. pure chloroform were manufactured per day in each still, in place of $70 \mathrm{lbs}$., which could be made by the old process, an increase in capacity of the plant of over roo per cent.

The amount of steam used by the new process was 2 tons for Ioo lbs. chloroform, against I. I tons in the process, for the same amount.

The same number of laborers ran the improved plant, after mechanical washers had been installed, the same laborers making more than twice the former product.

There are localities in the western states where corn, limestone and salt are cheap, where coal and power are abundant. There may be a market for caustic soda but none for bleach, which cannot be disposed of on account of the freight rates. By making chloroform, many pounds of material can be condensed into one and can be shipped to the centers of population.

\section{COMPARISON OF SOME METHODS FOR THE ESTIMATION OF SULFUR IN VULCANIZED RUBBER WITH ESPECIAL REFERENCE TO ELECTROLYTIC OXIDATION.}

\section{By D. Spence and J. Young.}

Received Feb. 3, 1912.

This work was primarily undertaken in order to find a rapid and, at the same time, accurate method for the determination of combined sulfur in vulcanized rubber, applicable to samples containing widely different quantities of combined sulfur. A method such as this is well-nigh essential to a study of the kinetics of vulcanization such as we had in view where a large number of simple compounds of rubber and sulfur containing all percentages of sulfur from 0.5 to 32 per cent. had to be investigated. Previous experience in the determination of the sulfur of vulcanization had shown that a method which might be applicable to one compound was not necessarily equally applicable to every other. This was a condition, however, which we had to meet in our work and it is one which is of importance in the every-day analysis of vulcanized rubber.

- In view of the ever-increasing importance of methods for the chemical analysis of India rubber and the attention which is being directed towards this subject by committees appointed here and abroad, this present work should be of interest not only as furnishing the solution to our specific problems, but as offering to those engaged in the analysis of India rubber a method for the determination of sulfur at once simple, rapid and accurate, which has not yet received that recognition in this country which it undoubtedly merits.

This is not, and does not pretend to be, a complete study of all the methods which have been published in the literature from time to time for the determina. tion of sulfur in India rubber. We have dealt here only with those methods which, from previous experience or from other reasons, seemed likely to meet the requirements of our work and our attention has, therefore, been confined to a comparative study of the methods of Carius, Henriques (methods involving fusion) and Gasparini (electrolytic oxidation) and to modifications of these by ourselves and others.

The Carius method requires no description here, It has long been recognized as an accurate one for the determination of sulfur in vulcanized rubber and we believe it is still in very general use where the highest accuracy is required. The disadvantages to the method are as well known, however, as the method itself. We need only refer, for example, to the cumbersome and altogether precarious nature of the work involved in the process which even the modifications investigated by us have not succeeded in eliminating. Similarly, the old method of Henriques ${ }^{x}$ for the determination of sulfur in rubber and the methods of Frank and Marckwald, 2 Wagner, ${ }^{3}$ and others, which are only slight modifications of the former, although perhaps simpler in some respects than the Carius method, require time and attention if reliable results are to be obtained, which, for our work at least, was out of the question. The fusion, namely, of the products of oxidation of the rubber by nitric acid, we have found to be so delicate and precarious an operation, even when carried out by the modified method described in the experimental part of this paper, that if constant care and attention is not given results of very uncertain value may be obtained. Our experience with methods of fusion indeed has been that in the long run more time is involved and less assurance of accuracy in the final result obtained than by a modified Carius method. There is little, if any, advantage, therefore, in fusion methods over Carius methods.

Far more interesting, however, than either of these methods is that of electrolytic oxidation. Of all methods for the estimation of sulfur in India rubber investigated by us, this method proved not only the simplest but also the most reliable. Not only is it unequaled in these respects, but we have found it applicable, and without modification, to the determination of the sulfur in rubber of any degree of vulcanization.

The method was originally evolved by Gasparinit 1 Z. angew. Chem., 34, 802 (1899).

${ }^{2}$ Gummi-Zeilung, 17, 71 (1903).

3 Ibid., 21, 552 (1907).

- Gaz. chim. ital., 37, II, 426-461 (1907). 
for the estimation of sulfur in organic compounds, including vulcanized rubber, and recently has been made use of by Hinrichsen ${ }^{2}$ in his work on vulcanization.

The method is an exceedingly accurate one. Indeed, in view of its simplicity and at the same time its accuracy, doing away as it does with all the objectionable features of other methods, it is surprising to us that it has not received more general application. We can only recommend it to the attention of those engaged in the analysis of vulcanized rubber.

To meet the case of complex rubber mixings the method is subject to the same modifications as are common to all existing methods.

In conjunction with a form of volumetric process for the determination of sulfur such as the barium chromate methodz investigated by us, or the benzidine method of Raschig, 3 we believe the process is capable of still further simplification to meet the requirements of a still more rapid and accurate method for the determination of sulfur in soft-cured rubber. Complex mixings would, of course, require special attention where the volumetric process using barium chromate is introduced. It is only, however, by practical work directed towards the solution of just such problems as these that real progress towards standard methods of analysis of rubber can be hoped to be made.

\section{EXPERIMENTAL PART.}

At the outset of this work it was necessary to adopt a standard method of analysis to which all our work could be referred. The Carius method was selected for this purpose in spite of the fact that the values obtained by it are stated to be slightly too hight on account of the inclusion in the precipitated sulfate of a very small quantity of silica dissolved from the glass. The error by this method we have found to be very small, however, and constant, if reasonable care is taken. In all our work, therefore, unless otherwise stated, our figures for sulfur are compared with those by the Carius method.

In carrying out the Carius method it has long been customary here to modify the original procedure to the extent of effecting the preliminary solution of the rubber in the acid in a closed bomb at as low a temperature as possible, afterwards opening the tube and heating it on the steam bath until further vigorous action ceases, then resealing the bomb and completing the oxidation by heating the tube to $170^{\circ}$ C. for 3 hours. In this way the intense gaseous pressure which is generated in a closed bomb by the first action of nitric acid on India rubber is relieved and the complete oxidation of the rubber takes place under conditions which, while preventing loss of sulfur, are less likely to bring disaster to the estimation by the bursting of the bomb in its final heating.

1 Koll. Zeitsch, , 8, 248 (1911). The statement by Waters and Tuttle, This Jovrnal, 3, 734 (1911), that this method is due to Hinrichsen is, therefore, incorrect. Gasparini has shown how the method may be applied to the oxidation of vulcanized rubber.

2 Amer. Chem. Jour., 2, 567 (1880).

3 . angew. Chem., 1903, 617 and 818. See Sutton's "Volumetric Analysis," 10th edit., p. 353 .

4 Anelli, Gaz. chim. ital., 41, I, 334.
In this modified form the Carius method has been found to be safer and less troublesome than the ordinary Carius process when applied, in particular, to samples of vulcanized rubber having a high content of rubber. The method has been tested by us against the standard Carius method, in which the rubber is first brought in contact with the acid in a closed bomb and is heated before the pressure is relieved, and has been found to give satisfactory results. A comparison of results obtained by this method against those obtained by the electrolytic process will also be found in the section on electrolytic oxidation, which follows.

In the modified method just described the Carius process still, however, remains tedious and somewhat precarious. To further simplify it an experiment was made in which the rubber was added directly but slowly, small pieces at a time, to the acid in the bomb which was warmed or cooled as required in order to complete the solution of the rubber without a too vigorous action ensuing. In this modification, therefore, the preliminary solution and heating took place in open bomb. A sample of hard rubber analyzed in this way gave 31.60 per cent. of sulfur as against $3^{1} .50$ per cent. by the ordinary Carius method. This method of treatment is somewhat simpler and apparently gives accurate results. Even with this modification, however, the Carius method is cumbersome where many sulfur estimations have to be made.

More promising, at first sight, as a rapid method for the determination of sulfur in vulcanized rubber, appears the process of fusion with alkaline oxidizing agents. This process, in its simplest form, consists in the fusion of an intimate mixture of the finely ground material with an excess of powdered sodium carbonate or a mixture of sodium carbonate with potassium nitrate. Such a method has recently been applied with success ${ }^{x}$ by one of us to the determination of bromine in the tetrabromide of India rubber and was found to give reliable and satisfactory results in this connection. The process is exceedingly simple. In our efforts to apply it to the determination of sulfur in vulcanized rubber we were confronted, however, with the difficulty of obtaining intimate mixture of the sample with the alkali fusion mixture. Sulfur values were always lower than those by the Carius method. Even in the case of a sample of finely powdered, hard rubber dust, where intimate mixture of the dust with the alkali was possible, $I 7.53$ per cent. of sulfur was found by this method as against 30.80 per cent. by the Carius method. One can only conclude from this that the chemical combination of rubber and sulfur to hard rubber is much more stable towards alkaline oxidizing agents than the corresponding bromine derivative of India rubber. Some incineration experiments carried out, using Eschka's mixture directly, proved equally unsuccessful for similar reasons.

The root difficulty in direct fusion methods as applied to vulcanized rubber undoubtedly lies in obtaining intimate mixture of the rubber material with

\footnotetext{
' Le Caowichouc et la Gutta Percha, 8, 88, 5313 (1911).
} 
the fusion mixture. It was to overcome this difficulty that Henriques ${ }^{I}$ evolved the method of first dissolving the rubber in nitric acid, taking the solution thus obtained to syrupy consistency before mixing with the fusion mixture. This is a great advance over simple fusion, and with care and attention the old method of Henriques has been found reliable in the hands of experienced analysts. For the sake of a comparison we made a number of estimations by the Henriques and our modified Carius method on samples of vulcanized rubber having widely different sulfur content. The Henriques method was carried out by us in the modified form as described by Frank and Marckwald. $x$ The results obtained by the two methods are given in tabular form in Table $\mathrm{I}$.

TABLE I.

Total Combined Total Combined Total Total. sulfur. sulfur. sulfur. sulfur. sulfur. sulfur. Per Per Per Per Per Per Method. cent. cent. cent. cent. cent. cent. $\begin{array}{lllllll}\text { Carius method.... } & 13.14 & 3.95 & 22.73 & 5.21 & 8.04 & 4.59\end{array}$ Henriques method., $\left.12.97 \quad 3.67\left\{\begin{array}{l}21.65 \\ 22.17 \\ 22.50\end{array}\right\} \begin{array}{l}5.08 \\ 5.05\end{array}\right\} \quad 7.97 \quad 4.44$

The results are not as satisfactory as we had hoped they would be. Our practical experience with the method, besides revealing to us that it is excessively cumbersome and altogether unsuitable for the work we had in view, showed that it still contains one cardinal weakness which the treatment with nitric acid in the first instance does not overcome. The treatment, namely, of the rubber by nitric acid results in the formation of nitro derivatives of the rubber ("nitrosites") which on fusion with alkali will sometimes cause spurting and small explosions with loss of sulfur even when the utmost care and constant attention is given to the fusion process. The results by the method are usually low in consequence. The trouble lies largely in the fact that it is well-nigh impossible to obtain a uniform mixture of the syrupy products of decomposition of the rubber by nitric acid with the solid alkaline mixture.

We lay no claim to be the first to draw attention to this inherent weakness in the Henriques fusion process. To overcome it, Wagner ${ }^{2}$ has suggested the diluting of the syrup obtained by treatment of the rubber by nitric acid with water, rendering the aqueous solution thus obtained faintly alkaline by means of solid $\mathrm{KOH}$ and afterwards concentrating to half bulk before mixing with excess of sodium carbonate and fusing. The underlying idea we believe to be a happy one. We have gone further, however, and have modified Henriques' method to the extent of mixing the products of oxidation of the rubber by nitric acid with the alkaline fusion mixture in solution, afterward taking the whole to dryness prior to fusing. In this way complete and thorough mixing of the products of oxidation of the rubber with the fusion mixture is effected and the subsequent fusion can be readily carried out without much difficulty or

1 Loc. cit.

2 Gummi-Zeituno, 21, 552 (1907). danger of loss due to ignition of the mixture. The method as carried out by us was as follows:

The rubber is dissolved in a mixture of ro cc. fuming $\mathrm{HNO}_{3}$, $5 \mathrm{cc} . \mathrm{H}_{2} \mathrm{O}$ and $3 \mathrm{~g}$. $\mathrm{KNO}_{3}$ with cautious warming, if necessary, to start the reaction. The solution obtained is evaporated almost to dryness, the residue dissolved in a solution of $6 \mathrm{~g}$. $\mathrm{Na}_{2} \mathrm{CO}_{3}$ in $\mathrm{I}_{5} \mathrm{cc} . \mathrm{H}_{2} \mathrm{O}$, thoroughly mixed with same, and again taken to dryness. The drying of the mixture and subsequent fusion arc carried out with the usual precautions:

$\begin{array}{ccc}\text { Modified Henriques. } & \begin{array}{c}\text { Carius. } \\ \text { Sulfur found. } \\ \text { Per cent. }\end{array} & \begin{array}{c}\text { Sulfur fouthd. } \\ \text { Per cent. }\end{array} \\ \text { Results. } & 31.26 & 30.80 \\ \text { I. } & 23.07 & 23.34 \\ \text { II. } & \end{array}$

That no great loss occurs in the process of fusion as carried out by us was shown by an experiment in which the rubber was dissolved in fuming nitric acid and water as above and the solution afterwards mado up to a known volume ( $5 \circ \mathrm{cc}$.) "with fuming nitric acid. One-half of this standard solution was treated by our modified Henriques method while the sulfur in the other portion was determined by Carius:

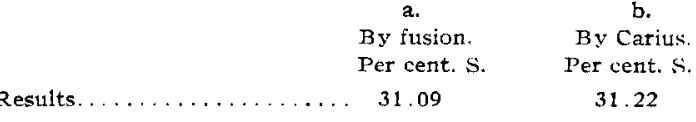

Some experiments on the modified Henriques method, using nitric acid saturated with bromine, as recommended by Waters and Tuttle, ${ }^{\text {T }}$ were also made.

The results are given in the following table and in comparison with the values by Carius are, on the whole, satisfactory:

\begin{tabular}{|c|c|c|c|}
\hline & $\begin{array}{l}\text { Sample I. } \\
\text { Combined s }\end{array}$ & $\begin{array}{l}\text { Sample II. } \\
\text { Combined s. }\end{array}$ & $\begin{array}{l}\text { Sample III. } \\
\text { Combined s }\end{array}$ \\
\hline Method. & Per cent. & Per cent. & Per cent. \\
\hline 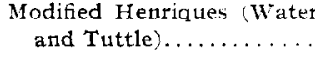 & 3.01 & 10.93 & 31.47 \\
\hline & 3.05 & 11.28 & 31.44 \\
\hline
\end{tabular}

The experience derived from our study of the methods of fusion outlined above showed, however, that although accuracy can be obtained by these methods, great care and constant attention is required if they are to be reliable in every instance. Even the best of them are cumbersome and are not adapted to rapid and at the same time accurate work. This is an important point where the accurate estimation of sulfur in India rubber is a routine laboratory operation.

Very different, on the other hand, were our experiences with the electrolytic process of decomposition. This process, originally designed by Gasparini ${ }^{2}$ for the estimation of sulfur in organic compounds in general, depends on the decomposition of the organic substance by nitric acid in presence of an electric current. Gasparini designed a somewhat complicated apparatus to carry out the oxidation of the organic substance without loss of sulfur. We have found, however, that as far as the sulfur of India rubber is concerned, any complication of this kind is unnecessary and that accuracy can be obtained by very simple means. In the early stages of our work on this method we were confronted, as was to be expected, with difficulties, difficulties not confined,

1 This Journal, 3, 10, 734 (1911).

${ }^{2}$ Loc. cit. 
however, to this method only, but these disappeared one by one as we became better acquainted with the method so that in the form in which we now present it, the method can be readily carried out even by the inexperienced.

The sample of rubber weighing about 0.5 gram $^{1}$ is put into a tall $200 \mathrm{cc}$. Jena glass beaker and covered with concentrated $\mathrm{HNO}_{3}$ (sp. gr. I.4). The beaker is covered by a clock glass and is then very gently warmed on the steam bath. As soon as the reaction starts the beaker is removed from the steam bath and the solution allowed to continue without further heating. If too much heat is applied at the beginning, it may be necessary to put the beaker in cold water in order to moderate the reaction. In about $2-3$ minutes the solution of the rubber is complete and all frothing of the liquid ceases. To this solution is now added $30 \mathrm{cc}$. of fuming nitric acid (sp. gr. I.5) with which the clock glass is washed. A current of electricity is now passed through the solution between electrodes of platinum foil. The most suitable current is 3 amperes with a potential of $6-8$ volts between the electrodes. The electrical connections are made as shown in the diagram (Fig. I).

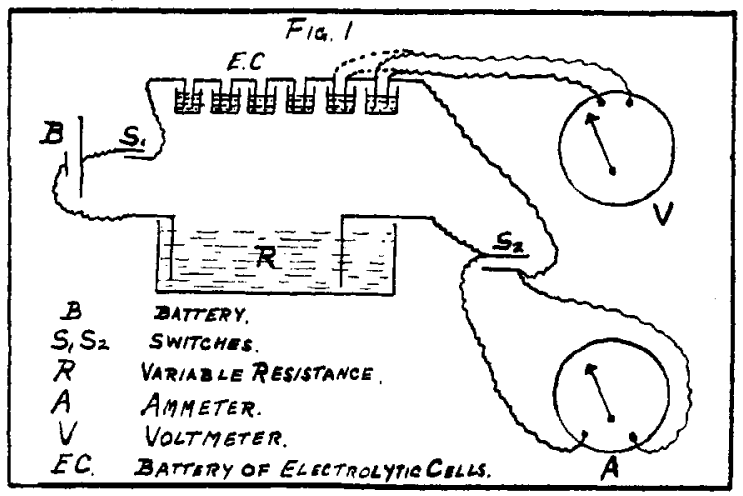

The current may be taken from any d. c. source and if necessary is reduced by means of a variable resistance, $R$. The ammeter $A$ is shunted at the terminals of the switch $S_{2}$ so that by opening the switch it is thrown into the circuit. The voltmeter $\mathrm{V}$ is introduced between the terminals of the electrodes. Any number of estimations may be connected up in series, the volt age in each cell being tested separately. The time required for the complete oxidation of the rubber varies with the sample. For soft-cured rubber containing a high percentage of rubber we have found that 2 to 3 hours are required. With hard rubbers containing a lower percentage of rubber the oxidation is complete in $3 / 4$ to $I$ hour. At the end of the action the electrodes are removed and washed with water. About one gram of pure $\mathrm{Na}_{2} \mathrm{CO}_{3}$ is then added and the solution evaporated to dryness on the steam bath. The residue is moistened with conc. $\mathrm{HCl}$ and taken to dryness again. The residue which should be free from nitric acid and nitrates is dissolved in hot water, filtered if necessary from insoluble matter and the sulfur precipitated as barium sulfate in the usual way.

The solution of the rubber, when carried out as described, proceeds smoothly and without difficulty. The electrolysis, when the current and voltage have once been determined, proceeds quietly and requires no attention whatsoever on the part of the operator. The final stage of the process is common to all methods for the determination of sulfur where nitric acid is used and requires no criticism here.

In order to still further simplify the method the

1 More may be conveniently used where the sulfur content of the sample is very low, apparatus shown in Fig. 2 was designed by.us. This

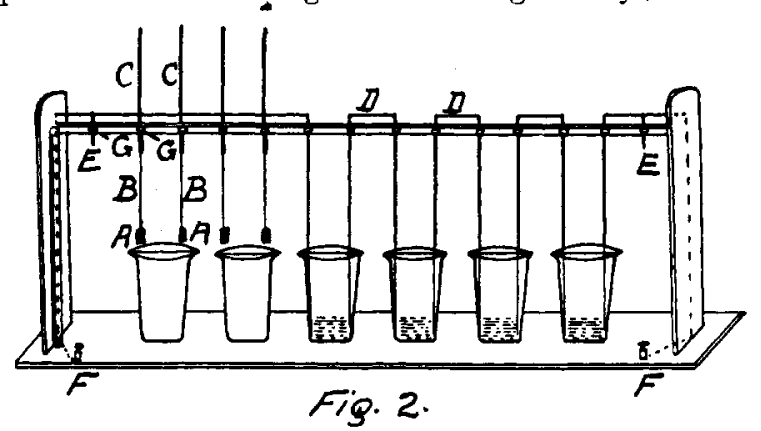

apparatus enables six estimations to be carried out at one time and does away with all binding screws and cumbersome connections-a very desirable feature, particularly where nitric acid is in constant use.

The decomposition of the sample is carried out in beakers as before. These are the regular "high form type" of beaker of Jena glass and have the following dimensions approximately: Height $100 \mathrm{~mm}$, diameter $55 \mathrm{~mm}$, capacity $200 \mathrm{cc}$. The beakers are covered by clock glasses split in two in order to admit of the wires carrying the electrodes. The electrodes $A$ are of platinum foil (one sq. inch in area) riveted to a piece of platinum wire, $B$. The free end of the wire is fused into a glass tube filled with mercury. The glass tubes slide in grooves cut in the cross support, and the electrodes can in consequence be fixed at any height by means of the wooden binding screws $\mathrm{G}, \mathrm{G}$. Connections are made by means of small bridges of copper wire, D, dipping into the mercury in the glass tubes. The binding screws $F, F$ are connected by means of a wire with the mercury cups $\mathrm{E}, \mathrm{E}$. With the connections arranged in this way it is a simple matter to remove or introduce an estimation at any stage, connection being made across the gap with a double-length bridge.

If the dimensions and quantities specified above are adhered to, a determination of the potential difference between the electrodes of each cell becomes unnecessary in each estimation, for, with the electrodes placed at the extreme circumference of the beaker, opposite one another and completely immersed in the acid, we have found that the required voltage is at once attained with sufficient accuracy for the purpose of the estimation.

The reaction in the cell, which is fairly vigorous at first giving rise to a considerable evolution of heat, soon moderates and when decomposition is complete almost ceases, the potential difference meantime falling gradually.

Some results of analyses of products of different degrees of vulcanization are given in the following table. These show the applicability of the method to the analysis of rubber of all degrees of vulcanization:

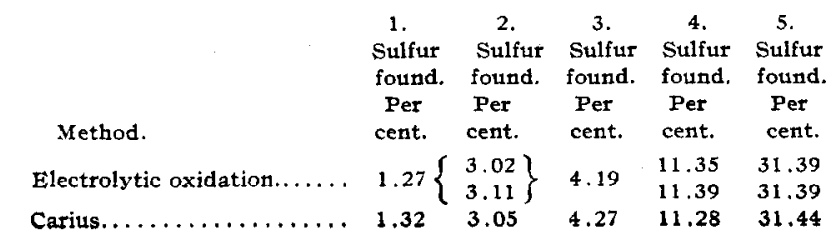


The method of electrolytic oxidation is now in regular use here. A great deal of work has recently been carried out by means of it where botk total and combined sulfur in vulcanized samples of rubber mixings had to be determined. These results have not only served to establish beyond all doubt the accuracy of the method but they have shown that it leaves little to be desired in the way of a simple process for the determination of sulfur in India rubber.

By introducing a volumetric process for the determination of the sulfur we have found it possible to still further simplify this method. We have found, namely, that the Pennock and Morton ${ }^{\mathrm{I}}$ rapid process for the determination of sulfur in coal and coke can be advantageously employed where the variation in the amount of sulfur present in different samples is not great.

This process depends on the decomposition of barium chromate by sulfuric acid, the liberation of iodine from potassium iodide in acid solution by the chromic acid set free and the titration of the iodine liberated by sodium thiosulfate. I cc. $N /$ IO thiosulfate is equivalent to 0.001067 gram sulfur.

A rigorous investigation of this process was first made on standard solutions of potassium sulfate of different concentration. We will refrain, however, from quoting our results in this connection. The method was found to be satisfactory where the sulfur present did not vary within wide limits. Several analyses of India rubber were then carried out by this method and checked against the ordinary gravimetric process. Results:

$\begin{array}{cc}\text { Volumetric. } & \text { Gravimetric. } \\ \text { Pet cent. S. } & \text { Per cent. S. } \\ 1.602 & 1.43 \\ 3.38 & 3.29 \\ 22.31 & 23.34\end{array}$

Only the results of the lower values are in agreement with the gravimetric figures. In carrying out this process one of the first essentials appears to be that the sulfuric acid and excess of barium chromate used in each experiment shall be as nearly as possible constant quantities. This is a comparatively easy matter to arrange where, as in the case of normal cured soft rubber, the sulfur does not usually exceed 5-6 per cent. and is a fairly constant quantity. But when, as in the examples cited, the variation is great and the correction to be applied is unknown and a constantly varying quantity, errors arise in the volumetric process. That it is impossible to overcome this difficulty by selecting a suitable weight of sample for analysis is fairly evident. This is not always an easy matter, however, where the sulfur content of the sample may vary between 0.5 per cent. and 32 per cent. In our work, therefore, in the meantime, we have adopted the gravimetric process until the foundations of the volumetric method shall have been more fully investigated.

\section{A METHOD FOR DETERMINING THE VALUE OF COMMER- CIAL STARCHES FOR USE IN COTTON MILLS. \\ BY G. M. MACNIDER. \\ Received Jan. 16, 1912. \\ In the manufacture of all grades of cotton cloth \\ 1 Jour. Am. Chem. Soc., 25, 1265.}

the yarn is prepared for the loom by a process known as sizing, in which it is treated with a solution of starch to give it certain properties essential to good weaving. With many grades of cloth, the cloth goes through a process known as finishing, where it is again treated with starch to produce a "finish" before it is put on the market. It is thus seen that the cotton mills use annually large quantities of starch and the purchase of the best starch for the purpose in hand becomes a very important matter.

The value of starch for cotton mill purposes depends on its property of swelling and forming a viscous solution when treated with hot water. It is the purpose of this paper to give a method for determining the viscosity of starch solutions which will show the comparative value of the different starches for use in cotton mills. The sizing and finishing of cotton goods are very technical operations and will not be taken up in detail. Sufficient reference will be made to them to show the function of the starch solution.

The object of sizing is to make the yarn stiffer, increase the strength and put it into proper condition for weaving. To accomplish this, the size must penetrate the yarn to some extent and also form a coating on the surface of the thread, which prevents wear of the thread in the loom. The size is prepared by boiling the starch (and other ingredients) with water and is applied to the yarn while hot. The machinery is so constructed that the threads are dried immediately after passing through the size.

The object of finishing is to give a stiffness to the cloth and produce a certain finish and "feel" which are very important factors in marketing cotton goods. As in sizing, the cloth is passed through a hot solution of the finish and then dried and rolled with hot rollers to bring out the finish. The use of the starch solution alone in these operations would make the goods too stiff and produce a harshness which is not desirable. To modify this, various softening agents are used, viz.; tallow, various oils, soaps, nagnesium chloride, magnesium sulfate, glycerine, etc., and in many mills various sizing and finishin prentions.

The principal starches used by the cott in mills are corn, potato, cassava, and to a small exwent wheat, sago and rice. In England, wheat flour and rice flutir are used to a considerable extent, but in America they are used very little. As will be seen later, each one of these starches has a different viscosity and therefore must be used in a different manner. Some authors attribute the differences in the characteristics of the starch solutions to the size and shape of the starch grains. There is no definite relation existing to prove this. The appearance of the different starch grains under the microscope are shown in the following cuts. ${ }^{2}$

A number of methods have been proposed for determining the viscosity of starch pastes, but in none of them has an attempt been made to determine the viscosity of the starch under conditions similar to those under which it is used in the cotton mills; conse-

2 These cuts were very kindly furnished the author by Mr. B. J. Howard, of the Bureau of Chemistry, Washington, D. C. 